ORIGINAL ARTICLE

\title{
Association between Skeletal Muscle Mass Index and Convalescent Rehabilitation Ward Achievement Index in Older Patients
}

\author{
Hideki Arai, MD a Naoya Ikegawa, PT a Masafumi Nozoe, PT, PhD ${ }^{\text {b }}$ Kuniyasu Kamiya, PT, PhD ${ }^{c}$ \\ and Satoru Matsumoto, MD a
}

\begin{abstract}
Objective: The aim of the current study was to investigate the association between the skeletal muscle mass index (SMI) and the convalescent rehabilitation ward achievement index (CRWAI) in older patients with functional impairment. Methods: We conducted a retrospective cohort study at a single rehabilitation center in Japan to include patients admitted to the convalescent rehabilitation ward because of neurological disease, motor disorder, or disuse syndrome. Patients with missing SMI data, those who died or were transferred to other hospitals due to comorbidities, those aged less than 65 years, and those hospitalized for $<7$ days were excluded from the study. We divided patients into two groups based on their SMI - the high SMI group (SMI $\geq 7.0 \mathrm{~kg} /$ $\mathrm{m}^{2}$ in men and SMI $\geq 5.7 \mathrm{~kg} / \mathrm{m}^{2}$ in women) and the low SMI group (SMI $<7.0 \mathrm{~kg} / \mathrm{m}^{2}$ in men and SMI $<5.7 \mathrm{~kg} / \mathrm{m}^{2}$ in women); we then evaluated the association between SMI and the CRWAI score. Results: Of the 319 recruited patients, 84 (26\%) were in the high SMI group. The medians and interquartile ranges of the CRWAI scores in the high SMI and low SMI groups were 38.6 (23.1-61) and $31.8(10.1-57.5)$, respectively $(\mathrm{P}=0.029)$. A high SMI was independently and negatively associated with the CRWAI score $(\beta=-0.16, \mathrm{P}=0.014)$. Conclusions: Our study showed that a high SMI was an independent factor negatively influencing the CRWAI score in older patients in a convalescent rehabilitation ward
\end{abstract}

Key Words: activities of daily living; convalescent rehabilitation; convalescent rehabilitation ward achievement index; sarcopenia; skeletal muscle mass index

\section{INTRODUCTION}

Loss of muscle mass and reduced muscle strength are the diagnostic features of sarcopenia. ${ }^{1)}$ Sarcopenia is an important risk factor for adverse outcomes, such as physical disability, poor quality of life, and death. ${ }^{1,2)}$ Sarcopenia thereby requires urgent attention, especially in rehabilitation hospitals where it is present in almost $50 \%$ of patients. ${ }^{3,4)}$ Yoshimura et al. reported that sarcopenia is associated with worsening of the recovery process for activities of daily living (ADLs) in hospitalized older adults receiving rehabilita- tion. ${ }^{3)}$ The latest consensus statement by the Asian Working Group for Sarcopenia (2019) emphasized that diagnosing sarcopenia requires the measurement of muscle mass, muscle strength, and gait speed. ${ }^{5)}$ However, it is sometimes not possible to measure muscle strength and gait speed in patients admitted to rehabilitation wards because of their physical disabilities. ${ }^{6}$ In such cases, it is important to measure the skeletal muscle mass index (SMI), which is one of the diagnostic criteria for sarcopenia. $\left.{ }^{4}\right)$

Evaluating the efficacy of rehabilitation interventions in the convalescent rehabilitation ward is critical in determining

Received: October 19, 2021, Accepted: December 28, 2021, Published online: January 29, 2022

a Toyonaka Heisei Hospital, Toyonaka, Japan

${ }^{b}$ Department of Physical Therapy, Faculty of Nursing and Rehabilitation, Konan Women's University, Kobe, Japan

${ }^{c}$ Department of Hygiene and Public Health, Osaka Medical and Pharmaceutical University, Takatsuki, Japan

Correspondence: Hideki Arai, MD, Toyonaka Heisei Hospital, 1-16-18 Haradanaka, Toyonaka-shi, Osaka 561-0807, Japan, E-mail:

deme216@yahoo.co.jp

Copyright (C) 2022 The Japanese Association of Rehabilitation Medicine

This is an open-access article distributed under the terms of the Creative Commons Attribution Non-Commercial No Derivatives (CC BY-NC-ND) 4.0 License. http://creativecommons.org/licenses/by-nc-nd/4.0/ 
its optimal functioning. ${ }^{7)}$ For this purpose, the convalescent rehabilitation ward achievement index (CRWAI) was introduced in convalescent rehabilitation in Japan in 2016. ${ }^{7)}$ The CRWAI is calculated once a month for the entire hospital, and a gain of $\geq 40$ points on the CRWAI indicates high efficacy of the convalescent rehabilitation process. ${ }^{8}$ However, to the best of our knowledge, the association between SMI and CRWAI has not been investigated. Therefore, we conducted a retrospective analysis to examine the association between the SMI and CRWAI score, and, secondarily, to evaluate the association between SMI and CRWAI $\geq 40$ in older patients admitted to the convalescent rehabilitation ward.

\section{MATERIALS AND METHODS}

\section{Study Design and Participants}

A retrospective cohort study was conducted at the Toyonaka Heisei Hospital, Japan, between April 1, 2019, and March 31, 2021, involving patients admitted to the convalescent rehabilitation ward because of neurological disease, motor disorders, or disuse syndrome. Disuse syndrome is a pathophysiological condition or group of symptoms that occurs after prescribed or unavoidable inactivity; the syndrome affect various body processes, including musculoskeletal, circulatory, and respiratory systems, thereby adversely affecting the performance of ADLs. ${ }^{9)}$ Patients with no SMI data, those who died or were transferred to other hospitals due to comorbidities, and those aged $<65$ years of age were excluded from the study. Additionally, patients hospitalized for $<7$ days were excluded because the effects of rehabilitation cannot be determined accurately within such short periods.

Following the definition of sarcopenia by the Asian Working Group for Sarcopenia, ${ }^{5)}$ we divided the patients into two groups: those with a high SMI (SMI $\geq 7.0 \mathrm{~kg} / \mathrm{m}^{2}$ in men and $\geq 5.7 \mathrm{~kg} / \mathrm{m}^{2}$ in women) and those with a low SMI (SMI $<7.0 \mathrm{~kg} / \mathrm{m}^{2}$ in men and $<5.7 \mathrm{~kg} / \mathrm{m}^{2}$ in women). We then evaluated the association between SMI and CRWAI.

The Research Ethics Committee of the Toyonaka Heisei Hospital approved this study in accordance with the Ethical Guidelines for Medical and Health Research involving Human Subjects in Japan (No. 202101). ${ }^{10)}$ As per the guidelines, written informed consent was replaced by the opt-out method.

\section{Convalescent Rehabilitation}

The rehabilitation protocols were planned for each patient according to Japan's medical insurance system. ${ }^{9)}$ Three 20min sessions each of physical therapy, occupational therapy, and speech/language therapy (nine sessions per day) were conducted for patients with neurological diseases ${ }^{9}$; three 20-min sessions each of physical therapy and occupational therapy (six sessions per day) were implemented for patients with motor disorders ${ }^{9}$; whereas, patients with disuse syndrome received three 20-min sessions each of physical therapy, occupational therapy, and speech-language therapy (a total of nine sessions per day). ${ }^{9}{ }^{9}$ The prescribed standard lengths of stay (LOS) in the convalescent rehabilitation ward for patients with a neurological disease without hyper brain dysfunction, with a neurological disease with hyper brain dysfunction, with motor disorder, and with disuse syndrome was $150,180,90$, and 90 days, respectively. ${ }^{9)}$

\section{Data Collection}

Basic information on all patients, which included age, sex, body mass index (BMI), oral feeding status at admission, the target disease for convalescent rehabilitation, the presence of chronic heart failure, the presence of chronic respiratory disease, and the presence of dementia, along with laboratory findings, were recorded at admission. Oral feeding was defined as a score of 4-7 on the Functional Oral Intake Scale. ${ }^{11)}$ The target diseases for convalescent rehabilitation were neurological disease without hyper brain dysfunction, neurological disease with hyper brain dysfunction, motor disorder, and disuse syndrome. The presence of chronic heart failure or chronic respiratory disease were ascertained from the medical records. Dementia was defined as a score of $\leq 20$ points on the Revised Hasegawa's Dementia scale or by the prescription of antidementia drugs. ${ }^{9,12,13)}$ Laboratory data included the levels of blood hemoglobin, creatinine, blood urea nitrogen, serum total protein, and serum albumin. Anemia was defined as hemoglobin levels $<13.0 \mathrm{~g} / \mathrm{dl}$ in men and $<12.0 \mathrm{~g} / \mathrm{dl}$ in women, as defined by the World Health Organization. ${ }^{9}$ 14)

Within $48 \mathrm{~h}$ of hospitalization, patients' hand-grip strength, skeletal muscle mass, and functional independence measure (FIM) scores for motor function (FIM-M) and cognitive function (FIM-C) were measured. The FIM evaluates a patient's independence level in performing $\mathrm{ADLs}^{11)}$; the FIM contains 18 items: 13 items are in the physical domain and 5 items relate to cognition. ${ }^{11)}$ Based on the level of independence, each item is scored from 1 to 7 , where 1 represents total dependence and 7 indicates complete independence. ${ }^{11)}$ The total FIM score ranges from 18 to 126 , of which the FIM-M score ranges from 13 to 91 points, and the FIM-C ranges from 5 to 35 points. ${ }^{11)}$ Lower scores indicate dependency in performing ADLs. Bilateral hand-grip strength 


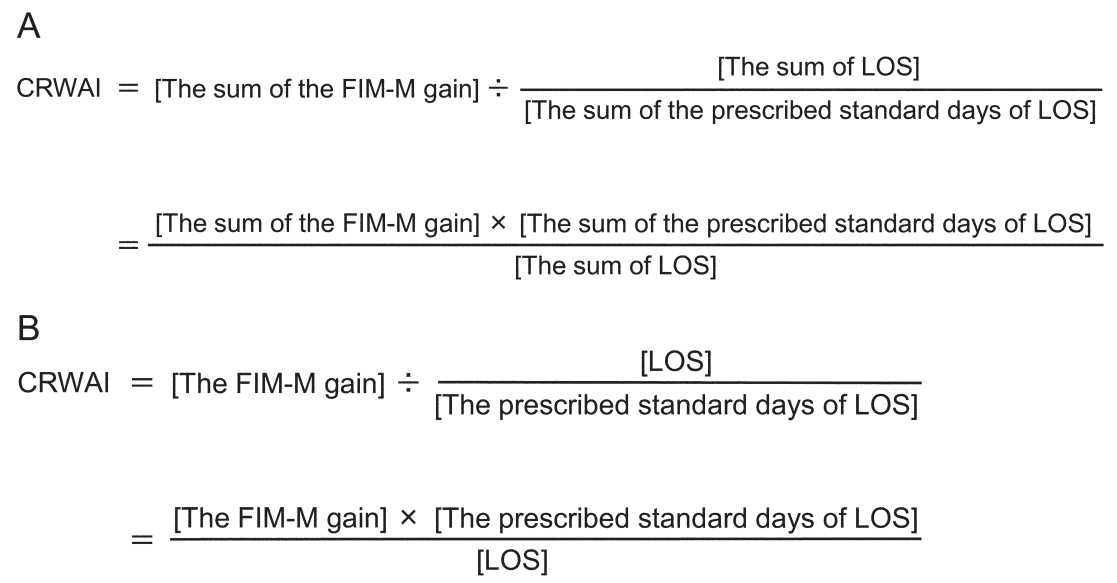

Fig. 1. (A) Calculation formula for CRWAI for all patients; (B) calculation formula for CRWAI for each patient.

was measured using a digital grip strength meter (Grip-D T.K.K.5401, Takei Kiki Kogyo, Niigata, Japan) with the patient in a standing or seated position, arms straight by the side; the highest value from three measurements was recorded. ${ }^{4)}$ For patients with hemiparesis, the hand-grip strength of the non-paralyzed hand was used. ${ }^{4)}$ Values of $<28 \mathrm{~kg}$ for men and $<18 \mathrm{~kg}$ for women were the thresholds for classifying the hand-grip strength as low; this is one of the diagnostic criteria for sarcopenia defined for use in Asia. ${ }^{5}$ ) Skeletal muscle mass was measured using bioelectrical impedance analysis (InBody S10, InBody, Tokyo, Japan) with subjects in the supine position. $\left.{ }^{4}\right)$ Skeletal muscle mass was not measured in patients with an implanted pacemaker and in those who could not remain relaxed during the measurement. SMI was calculated by dividing the measured appendicular muscle mass by the square of the height in meters. $\left.{ }^{4}\right)$ The FIM scores were measured again at discharge to calculate the final gain in FIM-M during hospitalization.

\section{Outcomes}

The primary outcome measures were the CRWAI score of each patient and whether each patient exceeded 40 points on the CRWAI; the latter is indicative of a highly efficient convalescent rehabilitation process. ${ }^{8)}$ CRWAI is calculated by dividing the sum of the FIM-M gains of all admitted patients at a given time by the sum of the adjusted LOS (which is calculated by dividing the sum of LOS by the prescribed standard days of LOS, e.g., 150 or 180 days in stroke case and 90 days in patients undergoing surgery for a femur fracture) (Fig. 1A) ${ }^{6)}$ Although the actual CRWAI is calculated for an entire hospital, the CRWAI score in this study was calcu- lated for each patient to allow comparisons between the two groups (Fig. 1B).

\section{Statistical Analysis}

We compared the clinical characteristics and outcomes in the high SMI and low SMI groups. Categorical data are presented as frequencies and percentages, whereas continuous data are given as means and standard deviations for parametric data or as medians and interquartile ranges for nonparametric data. The number of missing observed variables is also presented. We used the chi-squared test for group comparisons of categorical data, and, depending on the distributions of the data, we used the Student's $t$-test or Wilcoxon rank-sum test for between-group comparisons of continuous data .

Multiple linear regression analysis was used to determine whether SMI was independently associated with the CRWAI score. To adjust for bias, the following covariates were selected: age, sex, BMI, hand-grip strength, oral feeding at admission, neurological disease without hyper brain dysfunction, neurological disease with hyper brain dysfunction, motor disorder, disuse syndrome, chronic heart failure, chronic respiratory disease, dementia, baseline hemoglobin level, baseline serum albumin, FIM-M at admission, and FIM-C at admission. Multicollinearity was evaluated using the Variance Inflation Factor, where a value of 1-10 indicated the absence of multicollinearity.

We used logistic regression analysis to estimate the effects of outcomes of the high SMI group relative to the low SMI group. The results were reported as odds ratios (ORs) with 95\% confidence intervals (CIs). Clinically relevant variables 


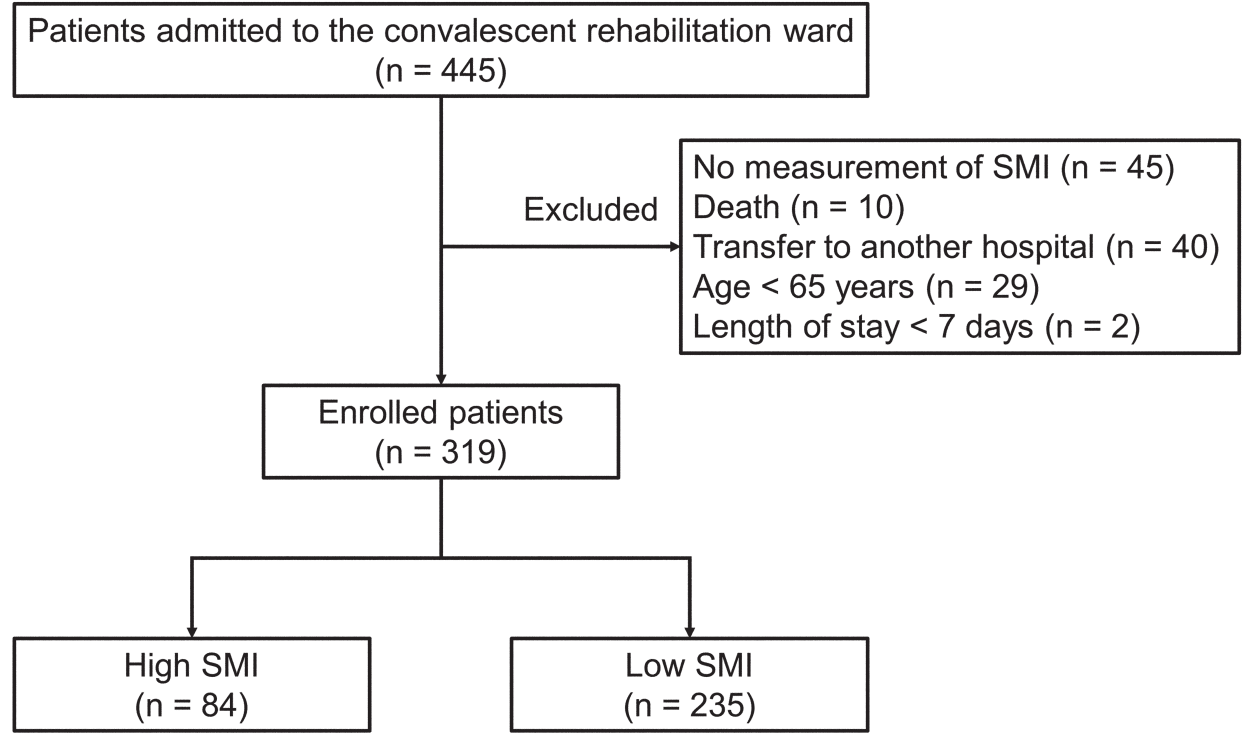

Fig. 2. Patient selection flowchart.

were adjusted for to estimate the adjusted OR in the multivariable logistic regression analysis. All the aforementioned covariates were adjusted for CRWAI exceeding 40 points. To assess the adjusted OR for each subgroup and the interaction through $\mathrm{P}$ values, we constructed the same multivariable logistic regression analysis for CRWAI $\geq 40$ in the subgroups. The subgroups of CRWAI $\geq 40$ included age $(\geq 75$ or $<75$ years), sex, BMI ( $\leq 18.5$ or $\left.>18.5 \mathrm{~kg} / \mathrm{m}^{2}\right)$, hand-grip strength, oral feeding at admission, neurological disease without hyper brain dysfunction, neurological disease with hyper brain dysfunction, motor disorder, disuse syndrome, chronic heart failure, chronic respiratory disease, dementia, anemia, and baseline serum albumin ( $\leq 3.5$ or $>3.5 \mathrm{~g} / \mathrm{dl})$.

All statistical analyses were performed using JMP 15 (SAS Institute, Cary, NC, USA). A two-sided $\mathrm{P}$ value of $<0.05$ was considered to indicate statistical significance.

\section{RESULTS}

\section{Patient Characteristics}

We initially registered 445 patients for this study, of which 126 (28\%) were excluded from the final analysis, leaving a study population of 319 cases (Fig. 2) with a mean age of 82 years; $116(36 \%)$ of the 319 participants were men (Table 1).

A total of $84(26 \%)$ patients were in the high SMI group; there were no statistically significant differences between the two groups in terms of age $(\mathrm{P}=0.06)$. $\mathrm{BMI}$ and hand-grip strength were significantly higher in the high SMI group than in low SMI (mean BMI, $23.8 \mathrm{~kg} / \mathrm{m}^{2}$ vs. $19.3 \mathrm{~kg} / \mathrm{m}^{2}, \mathrm{P}$
$<0.0001$; median hand-grip strength, $22 \mathrm{~kg}$ vs $15 \mathrm{~kg}$ in men, $\mathrm{P}=0.0005$; median, $14 \mathrm{~kg}$ vs $10 \mathrm{~kg}$ in women, $\mathrm{P}=0.0019$, respectively). Also, there were statistically significantly differences among the target diseases between the two groups $(\mathrm{P}=0.035)$. Compared with the low SMI group, the high SMI group had a higher proportion of patients receiving oral feeding at admission ( $94 \%$ vs. $81 \%, \mathrm{P}=0.0043$ ), and was less likely to have chronic heart failure ( $26 \%$ vs. $38 \%, \mathrm{P}=0.046)$, chronic respiratory disease ( $1 \%$ vs. $8 \%, \mathrm{P}=0.025)$, and dementia (48\% vs. $71 \%, \mathrm{P}=0.0001)$. Baseline hemoglobin and serum albumin levels were significantly higher in the high SMI group than in the low SMI group (mean $11.8 \mathrm{~g} / \mathrm{dl}$ vs. $11.3 \mathrm{~g} / \mathrm{dl}, \mathrm{P}=0.0077$; median $3.9 \mathrm{~g} / \mathrm{dl}$ vs. $3.6 \mathrm{~g} / \mathrm{dl}, \mathrm{P}=0.0004$, respectively). The median FIM and FIM-M scores were significantly higher in the high SMI group than in the low SMI group at admission (FIM: median 67 vs. 45, $\mathrm{P}<0.0001$; FIM-M: median 45 vs. 25, $\mathrm{P}<0.0001$, respectively; Table 1).

\section{Outcomes}

There was no statistically significant difference between gains in FIM and FIM-M scores between the two groups (for SMI high and low groups, respectively, median FIM gain: 26 vs 23, $\mathrm{P}=0.29$; median FIM-M gain: 22 vs $19, \mathrm{P}=0.37$ ). Likewise, the prescribed standard LOS days and the actual LOS between the high SMI group and low SMI groups were comparable (median prescribed standard LOS 90 days vs. 90 days, $\mathrm{P}=0.31$; median actual LOS: 64 days vs. 76 days, $\mathrm{P}=0.28$, respectively). However, the CRWAI score was significantly higher for the high SMI group (median=38.6 vs. 
Table 1. Patients' characteristics

\begin{tabular}{|c|c|c|c|c|}
\hline Variable & $\begin{array}{l}\text { All patients } \\
\qquad(n=319)\end{array}$ & $\begin{array}{l}\text { High SMI } \\
(\mathrm{n}=84)\end{array}$ & $\begin{array}{c}\text { Low SMI } \\
(\mathrm{n}=235)\end{array}$ & $\mathrm{P}$ value \\
\hline Age, years, mean (SD) & $82(7)$ & $80(7)$ & $82(7)$ & 0.06 \\
\hline Men, n (\%) & $116(36)$ & $29(35)$ & $87(37)$ & 0.68 \\
\hline Body mass index, kg/m², mean (SD) & $20.5(3.8)$ & $23.8(3.8)$ & $19.3(2.9)$ & $<0.0001$ \\
\hline \multicolumn{5}{|l|}{ Hand-grip strength, kg, median (IQR) * } \\
\hline Men & $17(11-22)$ & $22(16-26)$ & $15(10-21)$ & 0.0005 \\
\hline Women & $10(7-15)$ & $14(8-18)$ & $10(6-13)$ & 0.0019 \\
\hline Oral feeding at admission, n (\%) & $269(84)$ & $79(94)$ & $190(81)$ & 0.0043 \\
\hline \multicolumn{5}{|c|}{ The target disease for convalescent rehabilitation, $\mathrm{n}(\%)$} \\
\hline Neurological disease without HBD & $21(7)$ & $10(12)$ & $11(5)$ & \multirow{4}{*}{0.035} \\
\hline Neurological disease with HBD & $98(31)$ & $26(31)$ & $72(31)$ & \\
\hline Motor disorder & $102(32)$ & $30(36)$ & $72(31)$ & \\
\hline Disuse syndrome & $98(31)$ & $18(21)$ & $80(34)$ & \\
\hline \multicolumn{5}{|l|}{ Comorbidities, n (\%) } \\
\hline Chronic heart failure & $112(35)$ & $22(26)$ & $90(38)$ & 0.046 \\
\hline Chronic respiratory disease & $20(6)$ & $1(1)$ & $19(8)$ & 0.025 \\
\hline Dementia & $207(65)$ & $40(48)$ & $167(71)$ & 0.0001 \\
\hline \multicolumn{5}{|l|}{ Baseline laboratory data } \\
\hline Hemoglobin, g/dl, mean (SD) & $11.4(1.7)$ & $11.8(1.7)$ & $11.3(1.7)$ & 0.0077 \\
\hline Creatinine, mg/dl, median (IQR) & $0.7(0.6-1.0)$ & $0.8(0.6-0.9)$ & $0.7(0.6-1.0)$ & 0.54 \\
\hline Blood urea nitrogen, mg/dl, median (IQR) & $17.2(12.8-22.2)$ & $15.7(12-19.5)$ & $17.6(13.3-23)$ & 0.01 \\
\hline Total protein, g/dl, median (IQR) & $6.4(6.1-6.8)$ & $6.5(6.1-6.9)$ & $6.4(6.1-6.8)$ & 0.57 \\
\hline Albumin, g/dl, median (IQR) & $3.7(3.4-4.0)$ & $3.9(3.5-4.2)$ & $3.6(3.3-3.9)$ & 0.0004 \\
\hline \multicolumn{5}{|l|}{ FIM, median (IQR) } \\
\hline Total & $49(30-72)$ & $67(44-87)$ & $45(28-64)$ & $<0.0001$ \\
\hline Motor items & $29(16-45)$ & $45(23-57)$ & $25(15-40)$ & $<0.0001$ \\
\hline Cognitive items & $19(12-27)$ & $24(15-31)$ & $17(11-25)$ & 0.0003 \\
\hline
\end{tabular}

HBD, hyper brain dysfunction; IQR, interquartile range; SD, standard deviation.

* Variables with missing data - hand-grip strength: 3 in the high SMI group and 23 in the low SMI group.

31.8, $\mathrm{P}=0.029)$. In contrast, the proportion of patients with a CRWAI score $\geq 40$ was not significantly higher in the high SMI group than in the low SMI group ( $49 \%$ vs. $40 \%, \mathrm{P}=0.18$; Table 2).

There was no multicollinearity between the variables. Multiple linear regression analysis showed that SMI was independently and negatively associated with the CRWAI score $(\beta=-0.16, P=0.014)$. Oral feeding at admission, neurological disease without hyper brain dysfunction, and neurological disease with hyper brain dysfunction were all independently and positively associated with the CRWAI score $(\beta=0.13, P=0.037 ; \beta=0.14, P=0.023$; and $\beta=0.15, P=0.022$, respectively). The adjusted OR (95\% CIs) of the high SMI group relative to the low SMI group for CRWAI $\geq 40$ was 0.55 (0.26-1.16); adjusted OR (95\% CIs) values for men and hand-grip strength for CRWAI $\geq 40$ were $0.47(0.23-0.94)$ and 1.08 (1.02-1.15), respectively (Table 3).

In the subgroup analysis for CRWAI $\geq 40$, the upper boundaries of the $95 \%$ CIs were lower than 1 in the subgroups of patients without dementia and without anemia, and the interaction P value was significant for dementia (Fig. 3).

\section{DISCUSSION}

Our novel investigation revealed an independent and negative association between the SMI and the CRWAI score, but no significant association between SMI and CRWAI $\geq 40$. This study is the first to describe the effect of SMI on CRWAI in older patients admitted to a convalescent rehabilitation ward. 
Table 2. Comparison of data at discharge between the high SMI and low SMI groups

\begin{tabular}{lccc}
\hline Variable & $\begin{array}{c}\text { High SMI } \\
(\mathrm{n}=84)\end{array}$ & $\begin{array}{c}\text { Low SMI } \\
(\mathrm{n}=235)\end{array}$ & P value \\
\hline Gain in FIM, median (IQR) & & & \\
$\quad$ Total & $26(15-34)$ & $23(8-38)$ & 0.29 \\
$\quad$ Motor items & $22(13-30)$ & $19(6-33)$ & 0.37 \\
$\quad$ Cognitive items & $1(0-5)$ & $1(0-5)$ & 0.49 \\
The prescribed standard length of stay, days, median (IQR) & $90(90-180)$ & $90(90-180)$ & 0.31 \\
Actual length of stay, days, median (IQR) & $64(44-88)$ & $76(49-90)$ & 0.28 \\
CRWAI, median (IQR) & $38.6(23.1-61)$ & $31.8(10.1-57.5)$ & 0.029 \\
CRAWI $\geq 40, \mathrm{n}(\%)$ & $41(49)$ & $95(40)$ & 0.18 \\
\hline
\end{tabular}

Table 3. Multivariate analyses for the factors affecting CRWAI

\begin{tabular}{|c|c|c|c|c|c|c|c|}
\hline & \multicolumn{3}{|c|}{ CRWAI score } & \multicolumn{4}{|c|}{ CRWAI $\geq 40$} \\
\hline & $\beta$ & $\mathrm{P}$ & VIF & $\begin{array}{c}\text { Crude } \\
\text { ORs }\end{array}$ & $95 \%$ CIs & $\begin{array}{c}\text { Adjusted } \\
\text { ORs }\end{array}$ & $95 \%$ CIs \\
\hline High SMI & -0.16 & 0.014 & 1.59 & 1.47 & $0.89-2.43$ & 0.55 & $0.26-1.16$ \\
\hline Age & -0.046 & 0.45 & 1.3 & 0.95 & $0.92-0.98$ & 0.96 & $0.92-1.004$ \\
\hline Men & -0.066 & 0.34 & 1.7 & 0.86 & $0.54-1.36$ & 0.47 & $0.23-0.94$ \\
\hline BMI & 0.12 & 0.08 & 1.56 & 1.07 & $1.01-1.14$ & 1.03 & $0.94-1.12$ \\
\hline Hand-grip strength & 0.12 & 0.16 & 2.47 & 1.09 & $1.05-1.13$ & 1.08 & $1.02-1.15$ \\
\hline Oral feeding at admission & 0.13 & 0.037 & 1.36 & 2.75 & $1.38-5.49$ & 0.76 & $0.31-1.86$ \\
\hline Neurological disease without HBD & 0.14 & 0.023 & 1.3 & 2.85 & $1.12-7.26$ & 3.29 & $0.99-10.92$ \\
\hline Neurological disease with HBD & 0.15 & 0.022 & 1.63 & 0.61 & $0.37-0.99$ & 1.004 & $0.48-2.09$ \\
\hline Motor disorder & 0.011 & 0.87 & 1.6 & 2.04 & $1.27-3.29$ & 1.55 & $0.79-3.04$ \\
\hline Disuse syndrome & \multicolumn{3}{|c|}{ Reference } & \multicolumn{2}{|c|}{ Reference } & \multicolumn{2}{|c|}{ Reference } \\
\hline Chronic heart failure & -0.036 & 0.52 & 1.15 & 0.53 & $0.33-0.85$ & 0.67 & $0.38-1.19$ \\
\hline Chronic respiratory disease & -0.012 & 0.83 & 1.1 & 0.7 & $0.27-1.8$ & 0.68 & $0.22-2.13$ \\
\hline Dementia & -0.14 & 0.056 & 2.03 & 0.3 & $0.19-0.49$ & 0.96 & $0.46-2$ \\
\hline Hemoglobin & 0.025 & 0.7 & 1.46 & 1.11 & $0.98-1.27$ & 1.02 & $0.85-1.23$ \\
\hline Albumin & 0.067 & 0.35 & 1.87 & 3.7 & $2.08-6.57$ & 1.37 & $0.6-3.13$ \\
\hline FIM-M at admission & 0.13 & 0.14 & 2.56 & 1.04 & $1.02-1.05$ & 1.01 & $0.98-1.03$ \\
\hline FIM-C at admission & 0.04 & 0.66 & 2.96 & 1.09 & $1.06-1.12$ & 1.04 & 0.99-1.09 \\
\hline
\end{tabular}

CI, confidence interval; OR, odds ratio; VIF, variance inflation factor.

Covariates for CRWAI and adjusters for CRWAI $\geq 40$ : age, sex, BMI, hand-grip strength, oral feeding, neurological disease without HBD, neurological disease with HBD, motor disorder, disuse syndrome, chronic heart failure, chronic respiratory disease, dementia, baseline hemoglobin level, baseline serum albumin, FIM-M at admission, FIM-C at admission.

The CRWAI score for the high SMI group was significantly higher than that for the low SMI group, but high SMI was an independent and negative factor influencing the CRWAI score. A probable explanation for this seeming contradiction is that patients with high SMI did not have an advantage over patients with low SMI in terms of improvements in ADLs, a finding that was inconsistent with previous studies reporting that muscle mass is positively associated with ADLs of older patients. ${ }^{1,3,4,15)}$ There is a possibility that patients with high
SMIs had negative effects from other factors, such as reduced lower extremity range of motion, reduced lower limb muscle strength, and lower gait speed, than those with relatively low SMI; this was not assessed in the current study but has been reported to be correlated with ADLs. ${ }^{16,17)}$

In the current study, neurological disease and oral feeding at admission were independently and positively associated with the CRWAI score. The prescribed standard LOS for patients with a neurological disease (150 or 180 days) is 


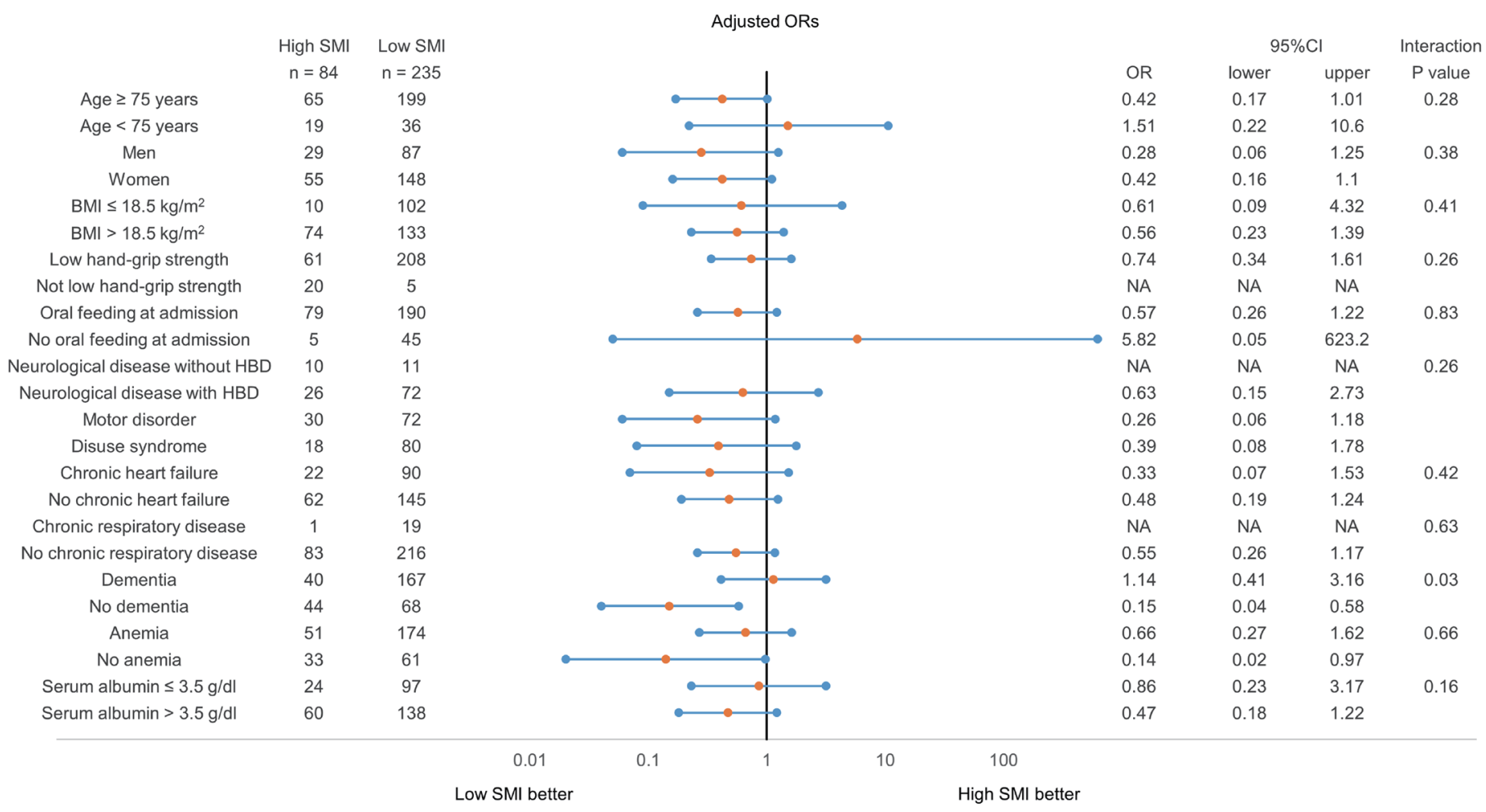

Fig. 3. Subgroup analysis on CRWAI $\geq 40$. NA, not assessed.

longer than that for patients with a motor disorder or disuse syndrome (90 days) ${ }^{9)}$; this fact may have proved advantageous when computing the CRWAI for patients with a longer prescribed standard LOS. Further, since dysphagia is reportedly associated with poorer functional recovery in older hospitalized patients, ${ }^{18)}$ patients on oral feeding may have had an advantage over those on tube feeding in improvement of ADLs.

Notably, there was no significant association between SMI and CRWAI $\geq 40$, but male gender and hand-grip strength were significantly associated with CRWAI $\geq 40$. Previous studies have reported that there are sex differences in the association between muscle mass and ADLs ${ }^{19,20)}$ and that appendicular skeletal muscle mass is higher in men than in women; however, the rate of decrease in appendicular skeletal muscle mass with age was significantly greater in men than in women. ${ }^{20,21)}$ Consequently, a loss of muscle mass is more likely to affect functional outcomes in older men than in older women. ${ }^{20)}$ Furthermore, several studies have reported an association of hand-grip strength with ADLs s $^{1,3,4,6,19,22-24)}$ that was retained for 1 year after hospital discharge in longitudinal studies. ${ }^{6}$ ) Therefore, hand-grip strength could be a good predictor of ADLs in patients admitted to rehabilitation wards. $1,3,4,6)$

\section{Limitations}

This study had several limitations. First, it was conducted at a single rehabilitation hospital; therefore, generalizing the results of this study should be done with caution. Future multicenter studies are needed to clarify the effect of SMI on the CRWAI. Second, we did not examine the interfering effect of various interventions, including rehabilitation treatment and nutrition therapy. Rehabilitation plays a major role in improving ADLs, and investigating the content of rehabilitation protocols is warranted in future research. Finally, there might be unexamined confounding factors in this retrospective study.

\section{CONCLUSIONS}

The current study showed that a high SMI is an independent factor that negatively influences the CRWAI scores in older patients in a convalescent rehabilitation ward. Further studies on the effect of SMI on CRWAI are needed to help improve the outcomes of convalescent rehabilitation.

\section{CONFLICTS OF INTEREST}

The authors report no conflicts of interest for this work. 


\section{REFERENCES}

1. Sánchez-Rodríguez D, Marco E, Miralles R, Fayos M, Mojal S, Alvarado M, Vázquez-Ibar O, Escalada F, Muniesa JM: Sarcopenia, physical rehabilitation and functional outcomes of patients in a subacute geriatric care unit. Arch Gerontol Geriatr 2014;59:39-43. DOI:10.1016/j.archger.2014.02.009, PMID:24726179

2. Sánchez-Rodríguez D, Calle A, Contra A, Ronquillo $\mathrm{N}$, Rodríguez-Marcos A, Vázquez-Ibar O, Colominas M, Inzitari M: Sarcopenia in post-acute care and rehabilitation of older adults: a review. Eur Geriatr Med 2016;7:224-231. DOI:10.1016/j.eurger.2015.11.001

3. Yoshimura Y, Wakabayashi H, Bise T, Nagano F, Shimazu S, Shiraishi A, Yamaga M, Koga H: Sarcopenia is associated with worse recovery of physical function and dysphagia and a lower rate of home discharge in Japanese hospitalized adults undergoing convalescent rehabilitation. Nutrition 2019;61:111-118. DOI:10.1016/j.nut.2018.11.005, PMID:30710883

4. Ohtsubo T, Nozoe M, Kanai M, Yasumoto I, Ueno K: Association of sarcopenia and physical activity with functional outcome in older Asian patients hospitalized for rehabilitation. Aging Clin Exp Res. [published online ahead of print July 13, 2021]. DOI:10.1007/ s40520-021-01934-8, PMID:34258733

5. Bai A, Xu W, Sun J, Liu J, Deng X, Wu L, Zou X, Zuo J, Zou L, Liu Y, Xie H, Zhang X, Fan L, Hu Y: Associations of sarcopenia and its defining components with cognitive function in community-dwelling oldest old. BMC Geriatr 2021;21:292. DOI:10.1186/s12877-02102190-1, PMID:33957882

6. Lunt E, Ong T, Gordon AL, Greenhaff PL, Gladman JR: The clinical usefulness of muscle mass and strength measures in older people: a systematic review. Age Ageing 2021;50:88-95. DOI:10.1093/ageing/afaa123, PMID:32706848

7. Sonoda S: Outcome indicators in comprehensive inpatient rehabilitation. Jpn J Compr Rehabil Sci. 2017;8:1-3.

8. Ministry of Health, Labour and Welfare: Outline of the revision of medical fees in 2020. [in Japanese] https:// www.mhlw.go.jp/content/12400000/000691039.pdf. Accessed 28 Sep 2021.
9. Arai H, Nozoe M, Matsumoto S, Morimoto T: Exercise training for patients with severe aortic stenosis in a convalescent rehabilitation ward - a retrospective cohort study . Circ Rep 2021;3:361-367. DOI:10.1253/ circrep.CR-21-0035, PMID:34250276

10. Ministry of Health, Labour and Welfare: Ethical guidelines for medical and health research involving human subjects. [in Japanese] https://www.mhlw.go.jp/ file/06-Seisakujouhou-10600000-Daijinkanboukouseikagakuka/0000080278.pdf. Accessed 30 Sep 2021.

11. Arai H, Takeuchi J, Nozoe M, Fukuoka T, Matsumoto S, Morimoto T: Association between active gait training for severely disabled patients with nasogastric tube feeding or gastrostoma and recovery of oral feeding: a retrospective cohort study. Clin Interv Aging 2020;15:1963-1970. DOI:10.2147/CIA.S270277, PMID:33116450

12. Matsumoto C, Ogawa H, Saito Y, Okada S, Soejima H, Sakuma M, Masuda I, Nakayama M, Doi N, Jinnouchi H, Waki M, Morimoto T, JPAD Trial Investigators: Sex difference in effects of low-dose aspirin on prevention of dementia in patients with type 2 diabetes: a longterm follow-up study of a randomized clinical trial. Diabetes Care 2020;43:314-320. PMID:31801787

13. Saw YM, Than TM, Win EM, Cho SM, Khaing M, Latt NN, Aung ZZ, Oo N, Aye HN, Kariya T, Yamamoto E, Hamajima N: Myanmar language version of the Revised Hasegawa's Dementia Scale. Nagoya J Med Sci 2018;80:435-450. PMID:30587859

14. Domenica Cappellini M, Motta I: Anemia in clinical practice - definition and classification: does hemoglobin change with aging? Semin Hematol 2015;52:261269. DOI:10.1053/j.seminhematol.2015.07.006, PMID:26404438

15. Nagano F, Yoshimura Y, Bise T, Shimazu S, Shiraishi A: Muscle mass gain is positively associated with functional recovery in patients with sarcopenia after stroke. J Stroke Cerebrovasc Dis 2020;29:105017. DOI:10.1016/j.jstrokecerebrovasdis.2020.105017, PMID:32807432

16. Jung H, Yamasaki M: Association of lower extremity range of motion and muscle strength with physical performance of community-dwelling older women. J Physiol Anthropol 2016;35:30. DOI:10.1186/s40101016-0120-8, PMID:27931244 
17. Mijnarends DM, Luiking YC, Halfens RJ, Evers SM, Lenaerts EL, Verlaan S, Wallace M, Schols JM, Meijers JM: Muscle, health and costs: a glance at their relationship. J Nutr Health Aging 2018;22:766-773. DOI:10.1007/s12603-018-1058-9, PMID:30080217

18. Matsuo H, Yoshimura Y, Ishizaki N, Ueno T: Dysphagia is associated with functional decline during acutecare hospitalization of older patients. Geriatr Gerontol Int 2017;17:1610-1616. PMID:27910255

19. Meskers CG, Reijnierse EM, Numans ST, Kruizinga RC, Pierik VD, van Ancum JM, Slee-Valentijn M, Scheerman K, Verlaan S, Maier AB: Association of handgrip strength and muscle mass with dependency in (instrumental) activities of daily living in hospitalized older adults -The EMPOWER Study. J Nutr Health Aging 2019;23:232-238. DOI:10.1007/s12603019-1170-5, PMID:30820510

20. Sawada M, Kubota N, Sekine R, Yakabe M, Kojima T, Umeda-Kameyama Y, Usami S, Akishita M, Ogawa S: Sex-related differences in the effects of nutritional status and body composition on functional disability in the elderly. PLoS One 2021;16:e0246276. DOI:10.1371/ journal.pone.0246276, PMID:33529213
21. Gallagher D, Visser M, De Meerrsman RE, Sepulveda D, Baumgartner RN, Pierson RN. Appendicular skeletal muscle mass: effect of age, gender, and ethnicity. J Appl Physiol 1985;83:229-239. DOI:10.1152/ jappl.1997.83.1.229, PMID:9216968

22. Wang DX, Yao J, Zirek Y, Reijnierse EM, Maier AB: Muscle mass, strength, and physical performance predicting activities of daily living: a meta-analysis. J Cachexia Sarcopenia Muscle 2020;11:3-25. DOI:10.1002/ jcsm.12502

23. Snih SA, Markides KS, Ottenbacher KJ, Raji MA: Hand grip strength and incident ADL disability in elderly Mexican Americans over a seven-year period. Aging Clin Exp Res 2004;16:481-486. DOI:10.1007/ BF03327406, PMID:15739601

24. Liu B, Chen X, Li Y, Liu H, Guo S, Yu P: Effect of passive finger exercises on grip strength and the ability to perform activities of daily living for older people with dementia: a 12-week randomized controlled trial. Clin Interv Aging 2018;13:2169-2177. DOI:10.2147/CIA. S174756, PMID:30464426 